\title{
Urogenital Abnormalities in Adenosine Deaminase Deficiency
}

\author{
Roberta Pajno ${ }^{1}$ - Lucia Pacillo ${ }^{2,3,4}$. Salvatore Recupero ${ }^{5,6}$ - Maria P. Cicalese ${ }^{5,6}$. Francesca Ferrua ${ }^{5,6}$. \\ Federica Barzaghi ${ }^{5,6}$ - Silvia Ricci ${ }^{7}$. Antonio Marzollo ${ }^{8}$ - Silvia Pecorelli ${ }^{9}$. Chiara Azzari ${ }^{7}$. Andrea Finocchi ${ }^{3,4}$. \\ Caterina Cancrini ${ }^{3,4}$ • Gigliola Di Matteo ${ }^{3,4}$. Gianni Russo ${ }^{1}$. Massimo Alfano ${ }^{10}$. Arianna Lesma ${ }^{11}$. \\ Andrea Salonia $^{10,12} \cdot$ Stuart Adams $^{13} \cdot$ Claire Booth $^{14}$. Alessandro Aiuti ${ }^{5,6,12}$
}

Received: 30 November 2019 / Accepted: 30 March 2020/Published online: 19 April 2020

(C) The Author(s) 2020

\begin{abstract}
Background Improved survival in ADA-SCID patients is revealing new aspects of the systemic disorder. Although increasing numbers of reports describe the systemic manifestations of adenosine deaminase deficiency, currently there are no studies in the literature evaluating genital development and pubertal progress in these patients.

Methods We collected retrospective data on urogenital system and pubertal development of 86 ADA-SCID patients followed in the period 2000-2017 at the Great Ormond Street Hospital (UK) and 5 centers in Italy. In particular, we recorded clinical history and visits, and routine blood tests and ultrasound scans were performed as part of patients' follow-up.

Results and Discussion We found a higher frequency of congenital and acquired undescended testes compared with healthy children (congenital, $22 \%$ in our sample, $0.5-4 \%$ described in healthy children; acquired, $16 \%$ in our sample, 1-3\% in healthy children), mostly requiring orchidopexy. No urogenital abnormalities were noted in females. Spontaneous pubertal development occurred in the majority of female and male patients with a few cases of precocious or delayed puberty; no patient presented high FSH values. Neither ADA-SCID nor treatment performed (PEG-ADA, BMT, or GT) affected pubertal development or gonadic function.

Conclusion In summary, this report describes a high prevalence of cryptorchidism in a cohort of male ADA-SCID patients which could represent an additional systemic manifestation of ADA-SCID. Considering the impact urogenital and pubertal abnormalities can have on patients' quality of life, we feel it is essential to include urogenital evaluation in ADA-SCID patients to detect any
\end{abstract}

Roberta Pajno, Lucia Pacillo, Claire Booth and Alessandro Aiuti contributed equally to this work.

Electronic supplementary material The online version of this article (https://doi.org/10.1007/s10875-020-00777-8) contains supplementary material, which is available to authorized users.

Alessandro Aiuti aiuti.alessandro@hsr.it

1 Department of Pediatrics, Endocrine Unit, IRCCS San Raffaele Scientific Institute, Via Olgettina, 60, 20132 Milan, Italy

2 Department of Pediatrics, "Pietro Barilla" Children Hospital, University of Parma, via Gramsci, 14 Parma, Italy

3 Unit of Immune and Infectious Diseases, Scientific Institute for Research and Healthcare (IRCCS) Childrens' Hospital Bambino Gesù, University Department of Pediatrics (DPUO), Rome, Italy

4 Department of Systems Medicine, University of Rome Tor Vergata, Rome, Italy

5 Pediatric Immunohematology and Stem Cell Program, IRCCS San Raffaele Scientific Institute, Via Olgettina, 60, 20132 Milan, Italy

6 San Raffaele Telethon Institute for Gene Therapy (SR-Tiget), IRCCS San Raffaele Scientific Institute, Milan, Italy
7 Division of Pediatric Immunology, Department of Health Sciences, University of Florence and Meyer Children's Hospital, Florence, Italy

8 Pediatric Hematology-Oncology Unit, Department of Women's and Children's Health, University of Padova, Via Giustiniani 3, 35128 Padova, Italy

9 Department of Pediatric Surgery, Ospedale dei Bambini - Spedali Civili, Brescia, Piazzale Spedali Civili 1, 25123 Brescia, Italy

10 Division of Experimental Oncology/Unit of Urology, URI, IRCCS Ospedale San Raffaele, Milan, Italy

11 Unit of Pediatric Surgery, Department of Urology, IRCCS Ospedale San Raffaele, Milan, Italy

12 Università Vita-Salute San Raffaele, Milan, Italy

13 SIHMDS-Haematology, Great Ormond Street Hospital for Children, London, UK

14 Department of Paediatric Immunology, Great Ormond Street Hospital, London, UK 
abnormalities, initiate early treatment, and prevent long-term complications.

Keywords ADA-SCID $\cdot$ puberty $\cdot$ pubertal development $\cdot$ cryptorchidism $\cdot$ undescended testis $\cdot$ urogenital abnormalities

\begin{tabular}{|c|c|}
\hline \multicolumn{2}{|c|}{ Abbreviations } \\
\hline $\mathrm{ADA}$ & Adenosine deaminase \\
\hline ADA-SCID & $\begin{array}{l}\text { Severe combined immunodeficiency } \\
\text { due to adenosine deaminase deficiency }\end{array}$ \\
\hline PEG-ADA & $\begin{array}{l}\text { Polyethylene glycol-conjugated } \\
\text { adenosine deaminase }\end{array}$ \\
\hline GT & Gene therapy \\
\hline BMT & Bone marrow transplantation \\
\hline $\mathrm{HH}$ & Hypogonadotropic hypogonadism \\
\hline $\mathrm{LH}$ & Luteinizing hormone \\
\hline FSH & Follicle-stimulating hormone \\
\hline
\end{tabular}

\section{Introduction}

Severe combined immunodeficiency due to adenosine deaminase deficiency is a rare autosomal recessive disease (ADASCID, OMIM \# 102,700) caused by mutations in the gene encoding the enzyme ADA type 1, resulting in impairment of the purine salvage pathway [1-3]. This defect in purine metabolism primarily affects lymphocyte development and function resulting in varying degrees of immune deficiency [4].

Several studies demonstrate that ADA-SCID is a systemic disease, and thanks to improved survival, an increasing number of non-immune manifestations are being recognized and reported [1-5].

At present, no study describes abnormalities in the development of genitalia or in the pubertal progression of ADASCID patients treated for their underlying immune disorder.

\section{Methods}

In this report, we describe data collected retrospectively on the urogenital system and pubertal development of 86 ADASCID patients followed in the period 2000-2017: 51 males and 35 females with an age range from 4 months to 30 years were included in this analysis (Table 1). Patients were from different ethnicities, and there was a high prevalence of consanguinity $(51 \%)$. Previous treatments included enzyme replacement therapy (PEG-ADA ERT), gene therapy (GT), or allogeneic bone marrow transplantation (BMT) as single therapy or given in various combinations (Table 1).

Patients in our cohort received immunological follow-up in five hospitals: 23 patients have been followed at our center, 1 patient at Bambin Gesù Hospital in Rome, 2 patients at Hospital Meyer in Florence, 1 patient at Hospital in Padova, and 59 patients in Great Ormond Street Hospital, London. Italian hospitals are part of the AIEOP (Associazione Italiana di Ematologia e Oncologia Pediatrica) and IPINET (Network Italiano Immunodeficienze Primitive) network.

Patients or their guardians provided written informed consent according to local consent procedures. This report was performed in accordance with the ethical standards of the institutional research committees and with the 1964 Helsinki declaration and its later amendments.

We collected the information registered during the immunological follow-up. Medical history, clinical data, routine blood tests, and ultrasound scans performed as part of patients' follow-up were recorded in patients' notes. If patients presented with clinical issues during the follow-up, additional investigations were performed. In male patients, we documented the number of patients with cryptorchidism, whether cryptorchidism was unilateral or bilateral, congenital (testis not present in the scrotum from birth by 3 months of age), or acquired (testis that was originally present in the scrotum at birth but ascends later) [6] or if the cryptorchidism solved spontaneously or required orchiopexy, the age of surgery, and any recurrences. We registered any urological malformation associated with cryptorchidism and the presence of phimosis and requirement for circumcision. Analyzing the complete cohort of patients, pubertal progression was evaluated at every clinical evaluation available for follow-up in both males and females. We documented the age of spontaneous puberty and every case of precocious or late puberty. Female patients underwent abdominal US scan as part of the follow-up; we documented data of any alteration of gonads at US scan. As markers of puberty, the following blood tests were performed in the majority of patients: luteinizing hormone (LH), follicle-stimulating hormone (FSH), testosterone (male patients), or estradiol (female patients). Analysis of these biomarkers (measured using fluorimetric methods) together with clinical evaluation of puberty allows evaluation of the hypothalamus-pituitary-gonad axis function. Moreover, if these hormones are evaluated in the first 3-6 months of life, it is possible to identify mini-puberty during which $\mathrm{LH}$ and FSH increase as it happens during puberty. This is a physiologic hormonal fluctuation without clinical manifestations associated with sex steroids rising to level reached in early-middle pubertal levels, without peripheral effects. If mini-puberty is identified with blood tests, it suggests normal hypothalamuspituitary-gonad axis function. It has been hypothesized that this hormonal phase has a role in physiologic descent of testis in the first year of life in transient congenital cryptorchidism [6-9]. 
Table 1 Sample description, sex, origin, parents' consanguinity, ADA-SCID treatment, and years of follow-up

$\mathrm{N}^{\circ}$ sex Origin $\quad \mathrm{C} \quad$ ADA mutation

Years of follow-

up $\S$ (age)

\begin{tabular}{|c|c|c|c|c|c|c|}
\hline 1 & $\mathrm{~F}$ & South America/Hispanic & Yes & $\begin{array}{l}\text { Compound heterozygous, c. } 320 \mathrm{~T}>\mathrm{C}, \mathrm{p} . \mathrm{L} 107 \mathrm{P} / \mathrm{c} .632 \mathrm{G}>\mathrm{A} \text {, } \\
\text { p.R211H }\end{array}$ & $\begin{array}{l}{\text { Haploidentical } \mathrm{BMT}^{\circ}}^{\rightarrow} \rightarrow \\
\text { GT }^{1} \rightarrow \text { PEG-ADA }\end{array}$ & $15(3-18$ y) \\
\hline 2 & M & South America/Hispanic & No & $\begin{array}{l}\text { Compound heterozygous, c. } 221 \mathrm{G}>\mathrm{T} \text {, p.G74V/c.845G }>\text { A, } \\
\text { p.R282Q }\end{array}$ & Haploidentical $\mathrm{BMT}^{\circ} \rightarrow \mathrm{GT}^{1}$ & $14(1-15 \mathrm{y})$ \\
\hline 3 & $\mathrm{~F}$ & Arabic/White & Yes & Homozygous c.845G > A, p.R282Q & $\begin{array}{l}\text { Haploidentical } \mathrm{BMT}^{\circ} \\
\text { PEG-ADA } \rightarrow \mathrm{GT}^{1}\end{array}$ & $13(1-14 \mathrm{y})$ \\
\hline 4 & $\mathrm{~F}$ & Arabic/White & Yes & $\begin{array}{l}\text { Compound heterozygous, c.646G > A, p.G216R/c.956_ } \\
\text { 960delAAGAG; p.E319GfsX3 }\end{array}$ & PEG-ADA $\rightarrow$ GT $^{1}$ & $11(1-12 \mathrm{y})$ \\
\hline 5 & M & Urope/White & Yes & Homozygous c. $632 \mathrm{G}>\mathrm{A}, \mathrm{p} . \mathrm{R} 211 \mathrm{H}$ & PEG-ADA $\rightarrow$ GT $^{1}$ & $12(5-17 \mathrm{y})$ \\
\hline 6 & $\mathrm{M}$ & Europe/White & No & $\begin{array}{l}\text { Compound heterozygous, c. } 646 \mathrm{G}>\mathrm{A}, \mathrm{p} . \mathrm{G} 216 \mathrm{R} / \mathrm{c} .872 \mathrm{C}>\mathrm{T} \text {, } \\
\text { p.S291L }\end{array}$ & PEG-ADA $\rightarrow$ GT $^{1}$ & $11(0-11 \mathrm{y})$ \\
\hline $\begin{array}{l}7 \\
8\end{array}$ & $\begin{array}{l}\mathrm{M} \\
\mathrm{F}\end{array}$ & $\begin{array}{l}\text { Europe/White } \\
\text { Arabic/White }\end{array}$ & $\begin{array}{l}\text { No } \\
\text { Yes }\end{array}$ & $\begin{array}{l}\text { Homozygous c. } 478+2 \mathrm{~T}>\mathrm{C} \\
\text { Homozygous c. } 646 \mathrm{G}>\mathrm{A}, \mathrm{p} . \mathrm{G} 216 \mathrm{R}\end{array}$ & $\begin{array}{l}\text { PEG-ADA } \rightarrow \text { GT }^{1} \\
\text { Haploidentical } \text { BMT }^{\circ} \rightarrow \\
\quad{\text { PEG-ADA } \rightarrow \text { GT }^{1}}^{2} \rightarrow\end{array}$ & $\begin{array}{l}10(1-11 \mathrm{y}) \\
8(0-8 \mathrm{y})\end{array}$ \\
\hline 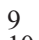 & M & outh America/Hispanic & Yes & Homozygous c.632G > A, p.R211H & PEG-ADA $\rightarrow$ GT $^{1}$ & $9(0-9 y)$ \\
\hline 10 & $\mathrm{M}$ & North America/White & No & $\begin{array}{l}\text { Compound heterozygous, c.646G > A, p.G216R/c.956_ } \\
\text { 960delAAGAG; p.E319GfsX3 }\end{array}$ & PEG-ADA $\rightarrow$ GT $^{1}$ & $9(1-10 \mathrm{y})$ \\
\hline 11 & M & South Asia & Yes & $\begin{array}{l}\text { Homozygous c. } 606+5 \mathrm{G}>\text { ? (Exon6, splice donor site }+5- \\
\text { no more data available) }\end{array}$ & PEG-ADA $\rightarrow$ GT $^{1}$ & $9(0-9 y)$ \\
\hline 12 & $\mathrm{M}$ & North America/White & No & $\begin{array}{l}\text { Compound heterozygous, c.646G > A, p.G216R/Exon10, } \\
\text { deletion + } 6 \text { c. } 975+6 \text { Tdel }\end{array}$ & PEG-ADA $\rightarrow$ GT $^{1}$ & $8(6-14 \mathrm{y})$ \\
\hline 13 & $\mathrm{~F}$ & Africa/White & No & Homozygous: c. $466 \mathrm{C}>\mathrm{T}$, p.R156C & PEG-ADA $\rightarrow$ GT $^{1}$ & $8(2-10 y)$ \\
\hline 14 & M & frica/Black & No & Homozygous, c.7C > T, p.Q3X & PEG-ADA $\rightarrow$ GT $^{1}$ & \\
\hline 15 & $\mathrm{M}$ & Africa/Black & Yes & Homozygous, c. $881 \mathrm{C}>$ A, p.T294K & $\underset{\text { PEGT }^{2}}{\text { BMA }^{2}} \rightarrow$ GT $^{1} \rightarrow$ MSD & $4(1-5 y)$ \\
\hline 16 & M & rabic/White & Yes & Homozygous, c.956_960delAAGAG, p.E319GfsX3 & PEG-ADA $\rightarrow$ GT $^{1}$ & $5(2-7 y)$ \\
\hline 17 & $\mathrm{~F}$ & European/White & No & $\begin{array}{l}\text { Compound heterozygous, c. } 632 \mathrm{G}>\mathrm{A}, \mathrm{p} . \mathrm{R} 211 \mathrm{H} / \mathrm{c} .646 \mathrm{G}>\mathrm{A} \text {, } \\
\text { p.G216R }\end{array}$ & PEG-ADA $\rightarrow$ GT $^{1}$ & $2(0$ \\
\hline 18 & M & Europe/Hispanic & No & $\begin{array}{l}\text { Compound heterozygous, c.467G > A, p.R156H / } \\
\text { c.646G > A, p.G216R }\end{array}$ & $\underset{\text { BMT }^{*}}{\text { PEG-ADA }} \rightarrow$ GT $^{1} \rightarrow$ MUD & $3(2-5 y)$ \\
\hline 19 & M & Europe/White & Yes & $\begin{array}{l}\text { Compound heterozygous, c. } 385 \mathrm{G}>\mathrm{A}, \mathrm{p} . \mathrm{V} 129 \mathrm{M} /(\text { second } \\
\text { mutation not identified) }\end{array}$ & PEG-ADA & $16(14-30 y$ \\
\hline 20 & $\mathrm{~F}$ & Europe/White & Unk & Homozygous, c.385G > A, p.V129M & PEG-ADA & $23(4-27 y)$ \\
\hline 21 & $\mathrm{~F}$ & $\mathrm{E}$ & No & s, c.499delG, pV167P & PEG & $8 \mathrm{y})$ \\
\hline 22 & M & Europ & Yes & Hom & PEG-ADA & $17(3-20 y)$ \\
\hline 23 & M & & Yes & $211 \mathrm{H}$ & PEG-ADA $\rightarrow$ MSD BMT $^{3}$ & $10(0-10 \mathrm{y})$ \\
\hline 24 & M & Europ & Unk & $>$ A, p.R211H & 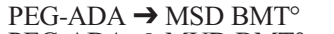 & $6(5-10 y)$ \\
\hline 5 & M & $\mathrm{ca} / \mathrm{Hispanic}$ & No & A A, p.R282Q & PEG-ADA $\rightarrow$ MUD BMT $^{\circ}$ & $1(0-1 \mathrm{y})$ \\
\hline 26 & $\mathrm{~F}$ & Europe/ & Unk & nore data available) & PEG-ADA $\rightarrow$ MSD BMT $^{\circ}$ & $14(0-14 \mathrm{y})$ \\
\hline 27 & $\mathrm{M}$ & Europe/White & No & $\begin{array}{l}\text { Compound heterozygous, c.466C > T, p.R156C/c.955 } \\
\text { 959GAAGA, p.E320GfsX3 }\end{array}$ & PEG-ADA $\rightarrow$ MUD BMT $^{\circ}$ & $13(1-14 \mathrm{y})$ \\
\hline 28 & $\mathrm{M}$ & White & Unk & ND & ИT* & $-15 \mathrm{y})$ \\
\hline 29 & $\mathrm{~F}$ & & Unk & & $\mathrm{A} \rightarrow \mathrm{MUD}$ BMT* & \\
\hline 30 & $\mathrm{~F}$ & South A & Unk & Home & $\mathrm{A} \rightarrow \mathrm{MSD}_{\mathrm{BMT}}^{\circ}$ & -13 y) \\
\hline 31 & $\mathrm{M}$ & Unk & Unk & C > T, p.R142X & $\mathrm{BMT}^{\circ}$ & $7 \mathrm{y})$ \\
\hline 32 & $\mathrm{~F}$ & Africa/Black & Yes & $\mathrm{C}>\mathrm{T}, \mathrm{p} . \mathrm{Q} 3 \mathrm{X}$ & 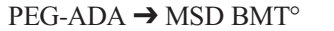 & $8 \mathrm{y})$ \\
\hline 33 & M & White Irish & Unk & $.646 \mathrm{G}>\mathrm{A}, \mathrm{p} . \mathrm{G} 216 \mathrm{R}$ & $\mathrm{BMT}^{\circ}$ & -17 y) \\
\hline 34 & $\mathrm{~F}$ & $\mathrm{~S}$ & Yes & ND & $\mathrm{A} \rightarrow \mathrm{MFD} \mathrm{BMT}^{\circ}$ & $18 \mathrm{y})$ \\
\hline 35 & $\mathrm{~F}$ & Europe/White & Unk & $\begin{array}{l}\text { Compound heterozygous, c. } 363-1 \mathrm{G}>\mathrm{C} / \mathrm{c} .364 \mathrm{G}>\mathrm{A} \text {, } \\
\text { p.G122R }\end{array}$ & PEG-ADA $\rightarrow$ MUD BMT $^{4}$ & $18(0-18 \mathrm{y})$ \\
\hline 36 & $\mathrm{M}$ & $\mathrm{E}$ & Yes & Homozygous, c.646G > A, p.G216R & PEG-ADA $\rightarrow$ MFD BMT ${ }^{\circ}$ & $7 \mathrm{y})$ \\
\hline 37 & $\mathrm{~F}$ & & & & $\mathrm{BMT}^{\circ}$ & 6 y) \\
\hline 38 & $\mathrm{~F}$ & te Irish & & $216 \mathrm{R}$ & $\mathrm{T}^{\circ}$ & \\
\hline 39 & $\mathrm{M}$ & & Yes & $\mathrm{H}$ & & $7 \mathrm{y})$ \\
\hline 40 & $\mathrm{M}$ & Af & & Q3X & $\rightarrow \mathrm{MFD} \mathrm{BMT}^{\circ}$ & $14(0-14 \mathrm{y})$ \\
\hline & M & & Yes & $216 \mathrm{R}$ & $\mathrm{P}$ & $13 \mathrm{y})$ \\
\hline & $\mathrm{F}$ & & & $\mathrm{H}$ & $\rightarrow$ MUD BMT $^{6}$ & $4 \mathrm{y})$ \\
\hline 43 & M & heritage & Yes & $16 \mathrm{G}>\mathrm{A}, \mathrm{p} . \mathrm{G} 239 \mathrm{D}$ & $\mathrm{A} \rightarrow \mathrm{MSD} \mathrm{BMT}^{\circ}$ & $2 \mathrm{y})$ \\
\hline 44 & $\mathrm{M}$ & Europe/White & No & $\begin{array}{l}\text { Compound heterozygous, c.367delG, p.D123TfsX10/c.956 } \\
\text { 960delAAGAG: p.E319GfsX3 }\end{array}$ & PEG-ADA $\rightarrow \mathrm{GT}^{5}$ & $13(0-13$ y) \\
\hline 45 & $\mathrm{~F}$ & Europe/White & No & $\begin{array}{l}\text { Compound heteroz } \\
\text { p.R156H/c.478 }\end{array}$ & $\begin{array}{l}\text { PEG-ADA } \rightarrow \text { GT }(\text { first })^{5} \rightarrow \\
\text { GT (second) }\end{array}$ & $13(2-15 \mathrm{y})$ \\
\hline 46 & $\mathrm{M}$ & & Yes & gous, c.7 & PEG-ADA $\rightarrow$ MSD BMT ${ }^{\circ}$ & \\
\hline 47 & $\mathrm{~F}$ & & & & $\mathrm{MT}^{\circ}$ & $2 \mathrm{y})$ \\
\hline 4 & M & & & $960 \mathrm{del} A$ & & \\
\hline 49 & $\mathrm{M}$ & Arabic & Yes & c. $385 \overline{\mathrm{G}}>\mathrm{A}, \mathrm{p}$ & $\rightarrow \mathrm{MSD} \mathrm{BMT}^{\circ}$ & \\
\hline 50 & M & & & ND & $\rightarrow \mathrm{GT}^{5}$ & $3(1-4 \mathrm{y})$ \\
\hline- & $\mathrm{F}$ & & $\mathrm{Y}$ & Hom & MSD Cord $^{\circ}$ & \\
\hline 52 & $\mathrm{M}$ & A & & $216 \mathrm{R}$ & PEG-AD & -11 y) \\
\hline 53 & $\mathrm{~F}$ & & Ys & $216 \mathrm{R}$ & PEG-ADA $\rightarrow$ MMUD Cord ${ }^{8}$ & $9(0-9 y)$ \\
\hline 51 & $\mathrm{~F}$ & & $\mathrm{Y}$ & Homo & PEG-ADA $\rightarrow$ MMUD Cord ${ }^{8}$ & $10(0-10 y)$ \\
\hline & M & & & $\mathrm{X} 28$ & $\mathrm{~A} \rightarrow$ MUD Cord ${ }^{8}$ & $3(0-3$ \\
\hline 56 & $\mathrm{M}$ & hite & $\mathrm{N}$ & $\begin{array}{l}\text { Compound heterozygous, c. } 466 \mathrm{C}>\mathrm{T} \text {, p.R } 156 \mathrm{C} / \mathrm{c} .646 \mathrm{G}>\mathrm{A} \text {, } \\
\text { p.G216R }\end{array}$ & PEG-ADA $\rightarrow \mathrm{GT}^{5} \rightarrow$ HSCT & $12(1-13 \mathrm{y})$ \\
\hline 57 & $\mathrm{M}$ & South Asia & Yes & Homozygous, c.646G > A, G216R & $\begin{array}{l}\text { PEG-ADA } \rightarrow \text { MUD Cord } \rightarrow \\
\text { MUD PBSC }\end{array}$ & $9(0-9 y)$ \\
\hline 30 & $\mathrm{~F}$ & & Yes & Hon & $\rightarrow \mathrm{MFD} \mathrm{BMT}^{\circ}$ & \\
\hline 59 & $\mathrm{M}$ & Eur & No & $\begin{array}{l}\text { Compound heterozygous, c. } 955-958 \text { delGAAG, } \\
\text { p.E320RfsX6/c.1078 + T >A }\end{array}$ & $\begin{array}{l}\text { PEG-ADA } \rightarrow \text { GT (first) } \rightarrow \\
\text { GT (second) }\end{array}$ & \\
\hline $\begin{array}{l}60 \\
61\end{array}$ & $\begin{array}{l}\mathrm{F} \\
\mathrm{M}\end{array}$ & $\begin{array}{l}\text { Arabic/White } \\
\text { Arabic/White }\end{array}$ & $\begin{array}{l}\text { Yes } \\
\text { Yes }\end{array}$ & $\begin{array}{l}\text { Homozygous, } 1079-15 \mathrm{~T}>\mathrm{A} \\
\text { Homozygous, c. } 385 \mathrm{G}>\mathrm{A} \text { p.V129M }\end{array}$ & $\begin{array}{l}\text { PEG-ADA } \rightarrow \text { MUD PBSC }^{5} \\
\text { PEG-ADA } \rightarrow \text { MFD BMT }^{10}\end{array}$ & 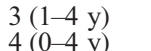 \\
\hline
\end{tabular}


Table 1 (continued)

\begin{tabular}{|c|c|c|c|c|c|c|}
\hline $\mathrm{N}^{\circ}$ & $\operatorname{sex}$ & Origin & $\mathrm{C}$ & ADA mutation & Treatment & $\begin{array}{l}\text { Years of follow- } \\
\text { up } \S \text { (age) }\end{array}$ \\
\hline 62 & M & Europe/White Irish & Yes & Homozygous, c.646G > A, p.G216R & PEG-ADA $\rightarrow$ MFD BMT ${ }^{0}$ & $7(0-7 \mathrm{y})$ \\
\hline 63 & $\mathrm{M}$ & Africa/Black & Yes & Homozygous, c.7C > T, p.Q3X & PEG-ADA $\rightarrow$ GT $^{7}$ & $3(4-7 y)$ \\
\hline 64 & M & South Asia & Yes & Homozygous, c.646G > A, p.G216R & PEG-ADA $\rightarrow$ GTT $_{7}^{7}$ & $7(0-7 y)$ \\
\hline 65 & $\mathrm{~F}$ & Europe/White & No & $\begin{array}{l}\text { Compound heterozygous, c.646G > A, p.G216R/c.955 } \\
\text { 959GAAGA, p.E320GfsX3 }\end{array}$ & PEG-ADA $\rightarrow \mathrm{GT}^{7}$ & $5(0-5 \mathrm{y})$ \\
\hline 66 & $\mathrm{~F}$ & South Asia & Yes & Homozygous, c.646G > A, p.G216R & PEG-ADA $\rightarrow \mathrm{GT}^{7}$ & $5(0-5 \mathrm{y})$ \\
\hline 67 & M & Arabic/white & No & Compound heterozygous, c.976-1G > C/c.302G > T, p.R101L & $\begin{array}{l}\text { PEG-ADA } \rightarrow \text { GT (first) }{ }^{7} \rightarrow \\
\text { GT (second) }\end{array}$ & $9(1-10 y)$ \\
\hline 68 & $\mathrm{M}$ & Africa/Black & No & Homozygous, c.7C > T, p.Q3X & PEG-ADA $\rightarrow \mathrm{GT}^{7}$ & $4(0-4 y)$ \\
\hline 69 & $\mathrm{~F}$ & Europe/White & No & $\begin{array}{l}\text { Compound heterozygous, c. } 872 \mathrm{C}>\mathrm{T} \text {, p.S291L/c. } 986 \mathrm{C}>\mathrm{T} \text {, } \\
\text { p.A329V }\end{array}$ & PEG-ADA $\rightarrow \mathrm{GT}^{7}$ & $4(1-5=y)$ \\
\hline 70 & M & Africa/Black & No & Homozygous: c.7C > T, p.Q3X & PEG-ADA $\rightarrow \mathrm{GT}_{7}^{7}$ & $4(0-4 y)$ \\
\hline 71 & $\mathrm{M}$ & Africa/Black & No & $\begin{array}{l}\text { Compound heterozygous, c. } 603 \mathrm{C}>\mathrm{G}, \mathrm{p} . \mathrm{Y} 201 \mathrm{X} / \mathrm{c} .632 \mathrm{G}>\mathrm{A} \text {, } \\
\text { p.R211H }\end{array}$ & PEG-ADA $\rightarrow \mathrm{GT}^{7}$ & $4(0-4 \mathrm{y})$ \\
\hline 72 & $\mathrm{~F}$ & Africa/Black & No & Homozygous, c.7C > T, p.Q3X & PEG-ADA $\rightarrow \mathrm{GT}^{7}$ & $4(9-13$ y) \\
\hline 73 & $\mathrm{~F}$ & Europe/White Irish & Yes & Homozygous, c.646G > A, p.G216R & PEG-ADA $\rightarrow \mathrm{GT}^{7}$ & $2(0-2 y)$ \\
\hline 74 & $\mathrm{M}$ & Europe/White & No & Homozygous, c.646G > A, p.G216R & $\begin{array}{l}\text { PEG-ADA } \rightarrow \text { MFD BMT } \rightarrow \\
\text { MSD BMT }^{\circ}\end{array}$ & $2(0-2 y)$ \\
\hline 75 & M & Unk & No & $\begin{array}{l}\text { Compound heterozygous, c. } 320 \mathrm{~T}>\mathrm{C} \text {, p.L107P/c. } 632 \mathrm{G}>\mathrm{A} \text {, } \\
\text { p.R211H }\end{array}$ & PEG-ADA $\rightarrow \mathrm{GT}^{7}$ & $1(1-2 \mathrm{y})$ \\
\hline 76 & $\mathrm{~F}$ & Africa/Black & Yes & Homozygous, c.7C > T, p.Q3X & PEG-ADA $\rightarrow \mathrm{GT}^{7}$ & $3(0-3 y)$ \\
\hline 77 & M & Europe/White Irish & No & Homozygous, c.646G > A, p.G216R & PEG-ADA $\rightarrow \mathrm{GT}_{7}^{7}$ & $2(0-2 y)$ \\
\hline 78 & $\mathrm{M}$ & Europe/White & Yes & $\begin{array}{l}\text { Compound heterozygous, c. } 310 \mathrm{C}>\mathrm{A}, \mathrm{p} . \mathrm{P} 104 \mathrm{~T} / \mathrm{c} .646 \mathrm{G}>\mathrm{A} \text {, } \\
\text { p.G216R }\end{array}$ & PEG-ADA $\rightarrow \mathrm{GT}^{7}$ & $2(0-2 y)$ \\
\hline 79 & $\mathrm{~F}$ & Europe/White & Yes & $\begin{array}{l}\text { Compound heterozygous, c. } 43 \mathrm{C}>\mathrm{G}, \mathrm{p} . \mathrm{H} 15 \mathrm{D} / \mathrm{c} .757_{-} \\
758 \text { dupCG }\end{array}$ & PEG-ADA $\rightarrow \mathrm{GT}^{7}$ & $2(0-2 y)$ \\
\hline 80 & $\mathrm{~F}$ & Europe/White & No & Homozygous, c. $646 \mathrm{G}>\mathrm{A}, \mathrm{p} . \mathrm{G} 216 \mathrm{R}$ & PEG-ADA $\rightarrow \mathrm{GT}_{7}^{7}$ & $2(0-2 y)$ \\
\hline 81 & $\mathrm{M}$ & Africa/White & No & Homozygous, c.704G > A, p.R235Q & PEG-ADA $\rightarrow$ GT $^{7}$ & $1(2-3 y)$ \\
\hline 82 & $\mathrm{M}$ & Europe/White Irish & Yes & Homozygous, c. $646 \mathrm{G}>\mathrm{A}, \mathrm{G} 216 \mathrm{R}$ & PEG-ADA $\rightarrow \mathrm{GT}_{7}^{7}$ & $2(0-2 y)$ \\
\hline 83 & $\mathrm{~F}$ & Europe/White & No & Homozygous, c. 320 T > C, p.L107P & PEG-ADA $\rightarrow \mathrm{GT}_{7}^{7}$ & \\
\hline 84 & M & Africa/Black & Yes & Homozygous, c.7C > T, p.Q3X & PEG-ADA $\rightarrow \mathrm{GT}_{7}^{7}$ & $1(0-1 \mathrm{y})$ \\
\hline 85 & $\mathrm{~F}$ & Europe/White Irish & Yes & Homozygous, c. $646 \mathrm{G}>\mathrm{A}, \mathrm{G} 216 \mathrm{R}$ & PEG-ADA $\rightarrow \mathrm{GT}_{7}^{7}$ & 0 \\
\hline 86 & $\mathrm{~F}$ & Europe/White-Africa/Black & No & $\begin{array}{l}\text { Compound heterozygous, c. } 482 \mathrm{G}>\mathrm{A}, \\
\text { p.W161X/c. } 1078+2 \mathrm{~T}>\mathrm{A}\end{array}$ & PEG-ADA $\rightarrow \mathrm{GT}^{7}$ & 0 \\
\hline
\end{tabular}

$C$ parents' consanguinity, Unk unknown, $\S$ years of follow-up are considered time from the first diagnostic test available to the last. In parentheses, age of the diagnostic test available-age of the last diagnostic test available. $N D$ not done, BMT bone marrow transplantation, GT gene therapy, $M S D$ BMT from matched sibling donor, $M F D$ BMT from matched family donor, $M U D$ BMT from matched unrelated donor, MMUD BMT from mismatched unrelated donor, PBSC peripheral blood stem cells, Cord cord blood cells

In the column treatment superscript numbers:

\footnotetext{
*Unknown

${ }^{0}$ No conditioning agents

${ }^{1}$ Busulfan (single agent, non myeloablative)

${ }^{2}$ Reduced toxicity regimen Treo/Flu

${ }^{3}$ Reduced intensity conditioning (RIC) Bu/Flu

${ }^{4}$ RIC Flu/Melph/ATG

${ }^{5}$ Melphalan (single agent)

${ }^{6}$ RIC Flu/Melph/Campath

${ }^{7}$ Low-dose busulfan (AUC 20)

${ }^{8}$ Myeloablative conditioning (MAC) Treo/Cy

${ }^{9} \mathrm{MAC}$ Treo/Flu

${ }^{10}$ Campath (single agent)
} 
Table 2 Male sample, urogenital abnormalities, pubertal development, hormonal tests, and testis US scan

\begin{tabular}{|c|c|c|c|c|c|c|c|c|}
\hline $\mathrm{N}^{\circ}$ & WG & CUT & AUT & Treatment of undescended testes & $\begin{array}{l}\text { Other urogenital } \\
\text { diseases }\end{array}$ & Puberty & $\begin{array}{l}\text { Testis structure at US } \\
\text { scan }\end{array}$ & $\begin{array}{l}\text { Hypothalamus- } \\
\text { pituitary- } \\
\text { gonads axis }\end{array}$ \\
\hline 2 & $\geq 37$ & Left & $\begin{array}{l}\text { Right } \\
\quad(9 \\
\text { y) }\end{array}$ & $\begin{array}{l}\text { Left orchidopexy } 2 \text { y and } 7 \text { months } \\
\text { Right orchidopexy } 9 \text { y and } 11 \text { months. } \\
\text { No relapse }\end{array}$ & Phimosis & Pubescent at 15 y (G2P4) & $\begin{array}{l}\text { Dyshomogeneous } \\
\text { (hyporeflectant } \\
\text { areas) since } 14 \mathrm{y}\end{array}$ & $\begin{array}{l}\text { Physiologic } \\
\text { activation }\end{array}$ \\
\hline 5 & 36 & Right & No & Right orchidopexy. No relapse & $\begin{array}{l}\text { 1) Phimosis } \\
\text { 2) inguinal hernia }\end{array}$ & Pubescent at 15 y G5 & Normal & $\begin{array}{l}\text { Physiologic } \\
\text { activation }\end{array}$ \\
\hline 6 & $36+1$ & Bilat & No & $\begin{array}{l}\text { Bilateral orchidopexy } 2 \text { y and } \\
3 \text { months. No relapse }\end{array}$ & Phimosis & Prepubescent at $10 \mathrm{y}$ & Normal & Not activated \\
\hline 7 & $\geq 37$ & No & No & NA & Phimosis & Prepubescent at $11 \mathrm{y}$ & ND & Not activated \\
\hline 9 & $\geq 37$ & No & $\begin{array}{l}\text { Right } \\
(6 \\
\text { y) }\end{array}$ & $\begin{array}{l}\text { Right orchidopexy } 7 \text { y and } 2 \text { months. } \\
\text { No relapse }\end{array}$ & Phimosis & Prepubescent at 9 y & $\begin{array}{l}\text { Hyporeflectant areas } \\
\text { since } 13 \mathrm{y}\end{array}$ & Not activated \\
\hline 10 & $\geq 37$ & no & No & NA & No & Prepubescent at $8 \mathrm{y}$ & ND & Not activated \\
\hline 11 & $\geq 37$ & Bilat & No & $\begin{array}{l}\text { Gonadoreline not effective. Bilateral } \\
\text { orchidopexy } 3 \text { y. Bilateral relapse } \\
\text { Gonadoreline not effective. Left } \\
\text { orchidopexy } 5 \text { y } 6 \mathrm{mo} \text {. Bilateral } \\
\text { relapse } 7 \text { y }\end{array}$ & $\begin{array}{l}\text { 1) Phimosis } \\
\text { 2) Micropenis } \\
\text { 3) Posterior } \\
\text { urethral valve } \\
\text { left megaureter }\end{array}$ & $\begin{array}{l}\text { Normal mini-puberty } \\
\text { Prepubescent at } 9 \mathrm{y}\end{array}$ & ND & Not activated \\
\hline 12 & 32 & no & No & NA & No & Prepubescent at $13 \mathrm{y}$ & $\begin{array}{l}\text { Homogenous but less } \\
\text { reflectant since } \\
11 \mathrm{y}\end{array}$ & Not activated \\
\hline 14 & $\geq 37$ & Left & $\begin{array}{r}\text { Bilat } \\
(7 \\
\mathrm{y})\end{array}$ & $\begin{array}{l}\text { Congenital undescended testes } \\
\text { spontaneously solved } \\
\text { Bilateral orchidopexy } 8 \text { y. No relapse }\end{array}$ & Phimosis & Prepubescent at $8 \mathrm{y}$ & Normal & Not activated \\
\hline 15 & $\geq 37$ & No & No & No & Phimosis & Prepubescent at $5 \mathrm{y}$ & $\begin{array}{l}\text { Homogenous but less } \\
\text { reflectant since } \\
11 \mathrm{y}\end{array}$ & Not activated \\
\hline 16 & $\geq 37$ & No & $\begin{array}{r}\text { Bilat } \\
(3 \\
\text { y) }\end{array}$ & $\begin{array}{l}\text { Bilateral orchidopexy } 4 y \text {. Left relapse } \\
5 \text { y }\end{array}$ & No & Prepubescent at $7 \mathrm{y}$ & Normal & Not activated \\
\hline 19 & $\geq 37$ & No & Right & $\begin{array}{l}\text { Gonadoreline, effective. Right relapse } \\
\text { right orchidopexy } \\
13 y+\text { hernioplastic. No relapse }\end{array}$ & Inguinal hernia & Pubescent at 30 y G5 & Normal & Not activated \\
\hline 22 & $\geq 37$ & No & $\begin{array}{r}\text { Left } \\
(2 \\
\mathrm{y})\end{array}$ & Spontaneously solved. No relapse & Inguinal hernia & Pubescent at 15 y G4 & ND & Not activated \\
\hline 24 & $\geq 37$ & No & $\begin{array}{r}\text { Bilat } \\
(5 \\
\text { y) }\end{array}$ & Bilateral orchidopexy 5 y. No relapse & No & $\begin{array}{l}\text { Pubescent- early onset (at } \\
11 \text { y G4) }\end{array}$ & $\begin{array}{l}\text { Hyperreflectant spots } \\
\text { (seminiferous } \\
\text { tubule fibrosis) }\end{array}$ & $\begin{array}{l}\text { Physiologic } \\
\text { activation }\end{array}$ \\
\hline 28 & Unk & No & No & NA & Micropenis & $\begin{array}{l}\text { CDGP; } 15 \text { y after } \\
\text { testosterone: G2P3A1 }\end{array}$ & Normal & ND \\
\hline 31 & $>37$ & Bilat & No & Unk & Micropenis & CDGP; at $17 \mathrm{y}: \mathrm{G} 3 \mathrm{P} 3 \mathrm{~A} 2$ & Normal & $\begin{array}{l}\text { Physiologic } \\
\text { activation }\end{array}$ \\
\hline 33 & Unk & No & No & NA & Micropenis & Unk & ND & ND \\
\hline 36 & $>37$ & No & No & NA & No & $\begin{array}{l}\text { CDGP; at } 17 \text { y after } \\
\text { testosterone: G4P4A2 }\end{array}$ & ND & ND \\
\hline 40 & $>37$ & No & No & NA & No & Prepubescent at $14 \mathrm{y}$ & Normal & ND \\
\hline 43 & $>37$ & Bilat & No & 2 y bilateral orchidopexy & No & $\begin{array}{l}\text { Pubescent at } 12 \text { y } \\
\text { (G4P3A2), early onset }\end{array}$ & ND & ND \\
\hline 57 & $>37$ & Bilat & No & Not done yet—performing follow-up & Micropenis & $\begin{array}{l}\text { Normal mini-puberty } \\
\text { Prepubescent at } 9 \mathrm{y}\end{array}$ & ND & ND \\
\hline 59 & $>37$ & Right & No & 11 y right orchidopexy & Phimosis & Prepubescent at $11 \mathrm{y}$ & ND & $\mathrm{ND}$ \\
\hline 62 & Unk & Left & No & 18 months left orchidopexy & No & Prepubescent at $7 \mathrm{y}$ & Normal & ND \\
\hline 68 & $>37$ & No & Right & 4 y right orchidopexy & No & $\begin{array}{l}\text { Prepubescent } 4 \mathrm{y} \text { and } \\
7 \text { months }\end{array}$ & Normal & Not activated \\
\hline 82 & $>37$ & Bilat & No & Not done yet—performing follow-up & $\begin{array}{l}\text { Micropenis } \\
\text { Undervirilized } \\
\quad \text { scrotum }\end{array}$ & $\begin{array}{l}\text { Suspect hypogonadism } \\
\text { hypogonadotropic (no } \\
\text { mini-puberty) } \\
\text { Prepubescent } 1 \text { y and } \\
7 \text { months }\end{array}$ & ND & $\mathrm{ND}$ \\
\hline
\end{tabular}

Only patients with urogenital abnormalities or alteration in puberty or patients who performed hormonal test/US testis scan are included in the table

Pubertal stage was evaluated with Tanner stage. Hypothalamus-pituitary-gonads axis evaluation: physiologic activation means we registered LH values $>1 \mathrm{mUI} / \mathrm{ml}$, FSH values $>2 \mathrm{mUI} / \mathrm{ml}$ and $<10 \mathrm{mUI} / \mathrm{ml}$, testosterone or estradiol levels adequate for age; not activated means LH values $<1 \mathrm{mUI} / \mathrm{ml}, \mathrm{FSH}$ values $<2 \mathrm{mUI} / \mathrm{ml}$, testosterone not detectable; normal mini-puberty means LH and FSH values similar to puberty values

$W G$ week gestation, $U T$ undescended testis, $N A$ not applicable, $N D$ not done, UNK unknown. Cryptorchidism: CUT congenital undescended testis, $A U T$ acquired undescended testis, in brackets the age of diagnosis, Bilat bilateral, $C D G P$ constitutional delay of growth and puberty 


\section{Results}

Regarding genital development, results differed between males and females.

Of 51 male patients, 11 (22\%) presented congenital undescended testes; of those, $6(54.5 \%)$ were bilateral and 7 $(63.6 \%)$ required orchidopexy, respectively (Table 2). Eight out of $51(16 \%)$ presented acquired undescended testes and among these $3 / 8$ were bilateral and $7 / 8$ required orchidopexy. None of the patients presenting with undescended testes were born at $<36$ weeks gestation. Six of 11 patients with congenital undescended testes had consanguineous parents $(54 \%$, Tables 1 and 2). Among other urogenital abnormalities seen, $3 / 51$ patients presented with inguinal hernia requiring surgical intervention, 6/51 presented micropenis of whom 4 had associated cryptorchidism, and one subject had posterior urethral valves. Nine out of $51(18 \%)$ presented phimosis, and 5/9 were treated with circumcision (Table 2).

Abdominal US scans performed in 10/35 female patients were normal with no abnormalities documented in ovaries, uterus, or vagina (Table 3).

In terms of pubertal development, data were available for 33 females and 48 males. In the overall population $28 / 81$ had achieved puberty and 52/81 are still prepubescent (aged less than 14 years). Among female patients, $51.5 \%$ are still prepubescent (age $\leq 10$ years) while $47.0 \%$ presented spontaneous pubertal progression (Table 3). Among these, $3 / 16$ presented early onset of puberty (at 8 years) and $2 / 3$ were treated with gonadotropin-releasing hormone agonists. Among the male patients (Table 2), $73 \%$ are still prepubescent (age $<14$ years).
Nine patients presented spontaneous pubertal development of whom 2 showed early onset of puberty (at 9 years). Three patients presented delayed onset of puberty but appropriate progression (constitutional delay in growth and puberty) of whom 2 were treated with testosterone inducing the onset of puberty.

Hormonal data are available in 20 patients (Table 4). In 9 pubescent patients ( 5 females, 4 males) hormonal tests showed physiologic activation of the hypothalamic-pituitarygonadal axis. In 11 prepubescent patients (11 males), LH, FSH, and testosterone or estradiol resulted low. None of the patients had raised FSH values. No patient with delayed puberty presented hypogonadotropic hypogonadism $(\mathrm{HH})$ although one patient with delayed puberty was not investigated (patient $\mathrm{n}^{\circ} 40$ - age 14 years). Three patients with micropenis and bilateral cryptorchidism underwent blood tests within the first 6 months of life (during mini-puberty), and 2 presented physiologic activation of hypothalamic-pituitary-gonadal axis (Table 2). In one patient, $\mathrm{HH}$ was suspected, and testosterone treatment was commenced (the patient is 1 year old).

\section{Discussion}

Currently, there are no studies in the literature evaluating genital development or pubertal progression in ADA-SCID patients. No abnormalities of the gonads, uterus, and vagina were detected in the female subgroup, even if these data should be taken with caution since only a minor proportion of female subjects was studied. Therefore, we cannot exclude

Table 3 Female sample, urogenital abnormalities at pelvic US scan, pubertal development, and hormonal tests

\begin{tabular}{|c|c|c|c|c|c|c|c|}
\hline $\mathrm{N}^{\circ}$ & $\begin{array}{l}\text { Pelvic us } \\
\text { scan }\end{array}$ & $\begin{array}{l}\text { Other urogenital } \\
\text { disease }\end{array}$ & Pubertal stage & $\begin{array}{l}\text { Precocious } \\
\text { puberty }\end{array}$ & $\begin{array}{l}\text { Treatment with GnRH } \\
\text { agonist }\end{array}$ & $\begin{array}{l}\text { Delayed } \\
\text { puberty }\end{array}$ & $\begin{array}{l}\text { Hypothalamus-pituitary- } \\
\text { gonads axis }\end{array}$ \\
\hline 3 & Normal & No & $\begin{array}{l}\text { Pubescent } 15 \text { y TS V } \\
\text { RM }\end{array}$ & No & No & No & ND \\
\hline 4 & Normal & No & $\begin{array}{l}\text { Pubescent } 12 \text { y TS V } \\
\text { RM }\end{array}$ & Yes $(8 \mathrm{y})$ & Yes (8-11 y) & No & ND \\
\hline 13 & Normal & No & $\begin{array}{l}\text { Pubescent } 10 \text { y, B4 } \\
\text { P2-3 }\end{array}$ & No & No & No & Physiologic activation \\
\hline 17 & Normal & No & Prepubescent 3 y & No & No & No & ND \\
\hline 20 & Normal & No & Pubescent RM & No & No & No & Physiologic activation \\
\hline 21 & Normal & No & Pubescent IM\# & Yes $(8 \mathrm{y})$ & No & No & Physiologic activation \\
\hline 42 & Normal & No & Pubescent $14 \mathrm{y}, \mathrm{RM}$ & No & No & No & Physiologic activation \\
\hline 45 & Normal & $\begin{array}{l}\text { Polycystic kidney } \\
\text { disease }\end{array}$ & $\begin{array}{l}\text { Pubescent at } 15 \mathrm{y}, \\
\text { RM }\end{array}$ & No & No & No & ND \\
\hline 53 & Normal & No & $\begin{array}{l}\text { Pubescent at } 9 \mathrm{y}, \\
\text { A1P2B3 }\end{array}$ & Yes $(9 \mathrm{y})$ & Yes (9 y-ongoing) & No & Normal \\
\hline
\end{tabular}

$N D$ not done, TS Tanner stage, $R M$ regular menses, $I M$ irregular menses

Only patients who performed US pelvic scan and/or hormonal tests were included in this table (for complete female sample see table in electronic supplemental material). In the column precocious puberty, the age of onset is reported in brackets

\# patient $\mathrm{n}^{\circ} 21$ presented irregular menstrual cycles with prolonged periods of amenorrhea associated with hyperinsulinism, hirsutism, and hyperandrogenism. Polycystic ovary syndrome was suspected, and the patient was treated with cyproterone acetate and transdermal estradiol 
Table 4 Puberty and hormonal tests in male and female patients

\begin{tabular}{|c|c|c|c|}
\hline $\mathrm{N}^{\circ}$ & Sex & Pubertal stage & Hypothalamus-pituitary-gonads axis \\
\hline 2 & M & Pubescent at 15 y (G2P4) & Physiologic activation \\
\hline 5 & M & Pubescent at 15 y (G5) & Physiologic activation \\
\hline 6 & M & Prepubescent at $10 \mathrm{y}$ & Not activated \\
\hline 7 & M & Prepubescent at $11 \mathrm{y}$ & Not activated \\
\hline 9 & M & Prepubescent at $9 \mathrm{y}$ & Not activated \\
\hline 10 & M & Prepubescent at $8 \mathrm{y}$ & Not activated \\
\hline 11 & M & Prepubescent at $9 \mathrm{y}$ & $\begin{array}{l}\text { Normal mini-puberty } \\
\text { Not activated }\end{array}$ \\
\hline 12 & M & Prepubescent at $13 \mathrm{y}$ & Not activated \\
\hline 13 & $\mathrm{~F}$ & Pubescent 10 y, B4 P2-3 & Physiologic activation \\
\hline 14 & M & Prepubescent at $8 \mathrm{y}$ & Not activated \\
\hline 15 & M & Prepubescent at $5 \mathrm{y}$ & Not activated \\
\hline 16 & M & Prepubescent at $7 \mathrm{y}$ & Not activated \\
\hline 19 & M & Pubescent at 30 y (G5) & Physiologic activation \\
\hline 20 & $\mathrm{~F}$ & Pubescent, regular menses & Physiologic activation \\
\hline 21 & $\mathrm{~F}$ & $\begin{array}{l}\text { Pubescent, irregular menses } \\
\text { (polycystic ovary syndrome) }\end{array}$ & Physiologic activation \\
\hline 24 & $\mathrm{M}$ & Pubescent at 10 y (G1-2) & Physiologic activation \\
\hline 42 & $\mathrm{~F}$ & Pubescent 14 y, regular menses & Physiologic activation \\
\hline 53 & $\mathrm{~F}$ & Pubescent at 9 y: A1P2B3 & Physiologic activation \\
\hline 57 & M & Prepubescent at $9 \mathrm{y}$ & $\begin{array}{l}\text { Normal mini-puberty } \\
\text { Not activated }\end{array}$ \\
\hline 82 & M & Prepubescent 1 y and 7 months & $\begin{array}{l}\text { Suspected hypogonadotropic hypogonadism, } \\
\text { no mini-puberty (testosterone treatment) }\end{array}$ \\
\hline
\end{tabular}

Only patients who performed hormonal tests were included in this table. Pubertal stage evaluated with Tanner stage. Hypothalamus-pituitary-gonads axis evaluation: physiologic activation means we registered LH values > $1 \mathrm{mUI} / \mathrm{ml}$, FSH values $>2 \mathrm{mUI} / \mathrm{ml}$ and $<10 \mathrm{mUI} / \mathrm{ml}$, testosterone or estradiol levels adequate for age; not activated means $\mathrm{LH}$ values $<1 \mathrm{mUI} / \mathrm{ml}, \mathrm{FSH}$ values $<2 \mathrm{mUI} / \mathrm{ml}$, testosterone or estradiol not detectable; normal mini-puberty means LH and FSH values similar to puberty values the association of urogenital abnormalities in female ADASCID. Conversely, we identified a high proportion of congenital and acquired undescended testes. In particular, the incidence of congenital undescended testes was higher in our cohort $(22 \%)$ compared with healthy full-term neonates (0.5-4\%, few authors report incidence up to 9\%) [6-8]. Moreover, while in the general population $70-80 \%$ of undescended testes resolve spontaneously with only $23 \%$ requiring orchidopexy, the proportion of ADA-SCID patients eventually requiring orchidopexy was higher, with $64 \%$ of finally requiring surgery.

A higher incidence of congenital undescended testes is detected in premature neonates (up to $45 \%$ ) $[6,7]$ but all patients with cryptorchidism in our sample were born at term (Table 2). Congenital cryptorchidism is a manifestation of numerous clinical syndromes; the ratio of non-syndromic to syndromic cryptorchidism is described to be greater than $6: 1$ [7]. In our sample there is high percentage of consanguinity (54\% of patients with congenital undescended testes have consanguineous parents, Tables 1 and 2). Given the high rate of consanguinity in our cohort we cannot rule out the possibility of an additional inherited defect accounting for this increased incidence. However, even in patients without consanguineous parents, the incidence remains high compared with the general population $(5 / 51,10 \%)$.

Considering the pathogenesis, cryptorchidism is due to aberrant embryological development. The embryology of testicular descent is complex involving numerous anatomical structures and hormones [6-7]. Androgens are known to play a role in this as $\mathrm{HH}$ and panhypopituitarism are associated with bilateral cryptorchidism [9]. Also, the possibility that environmental chemicals interfere with normal reproductive tract development has been raised [7]. We feel we can exclude the hypothesis of $\mathrm{HH}$ here as we did not detect a delay in puberty usually associated with $\mathrm{HH}$. Thirty-five percent of our patients entered spontaneous pubertal development and progression with adequate hormone levels; the remaining patients are aged 14 years or less. One can hypothesize that ADA may play a role in testicular embryological development/descent, and/or it is possible that toxic purine metabolites could interfere with this process. 
In our population, we also identified a high incidence of acquired undescended testis (16\%), with $87 \%$ of cases requiring orchidopexy. In a healthy population, acquired undescended testes are reported to occur in 1-3\% of cases [8]. Acquired undescended testes have a different pathogenesis compared with congenital undescended testes [7], mainly related to adhesions or increased stiffness/shortness of anatomical barriers involved. It is possible that metabolic abnormalities related to ADA deficiency could alter the histologic structure of these tissues. The toxic effect of ADA metabolites has been reported on different tissues, and it is well described how purinergic signaling plays an important role in fibrosis damage of several organs (skin, heart, liver, and lung) during tissue repair. For example, the profibrotic role of ADA deficiency in the lung has been clearly shown in an animal model with adenosine deaminase-deficient mice developing adenosine-dependent pulmonary fibrosis due to accumulation of ADA metabolites $[10,11]$. We can hypothesize that ADA deficiency could cause fibrosis in tissues that are crossed by testes, increasing the stiffness of the physiologic anatomical barriers.

In our patients receiving PEG-ADA ERT, BMT, or GT (with or without conditioning), FSH was not elevated. Thus, in our sample, neither ADA deficiency nor the treatments received negatively affected pubertal development or gonadic function. We did not perform specific tests to evaluate fertility in our cohort, mainly due to the young age of the patients. We can assume that our patients have functional endocrine regulation of puberty as they have normal pubertal development and normal testosterone or estradiol levels. The oldest patient is 30 years, but the mean age of the group is 19 years. However, we cannot know whether a dysfunction of endocrine gonadal component will have a later onset. No data are available in the literature regarding fertility in ADA-SCID. For patients undergoing BMT, there is a risk of infertility which of infertility is higher (>80\%) in patients treated with conditioning regimens containing TBI, high-dose cyclophosphamide, melphalan, and busulfan. The use of a reduced-intensity conditioning regimen is expected to decrease HSCT-related side effects. Recently, the Pediatric Diseases Working Party of the European Society for Blood and Marrow Transplantation has established recommendations for the diagnosis and pre-emptive procedures that should be offered to all children and adolescents in Europe who undergo life-saving allogeneic SCT [12]. Emerging reports describe fertility and gonadal function in transplanted SCID [13-15], but actually, no specific studies on ADA-SCID have been performed. We recommend that these aspects deserve special attention considering the systemic manifestations of the condition (ADA-SCID) and the potential effects of its treatments on gonadal function.

In the literature, excess of adenosine in murine penile erectile tissues has been described associated with priapism [16]: This study highlights how adenosine deaminase plays a biological role in different tissues and systems. Considering our sample's age, we did not analyze the erectile dysfunction.
The major limit of this report is the number of patients evaluated: We recognize that this study is based on limited sample size, but it is expected considering that ADA-SCID is an ultra-rare disease (from 1:200,000 to 1:1,000,000 births).

\section{Conclusion}

In summary, this report describes the high incidence of urogenital abnormalities in a cohort of male ADA-SCID patients, which likely represents systemic manifestations of ADA-SCID. We identified a high incidence of cryptorchidism in our male patients with no urogenital abnormalities noted in females. Spontaneous and age appropriate pubertal development occurred in most females and males with a few cases of precocious or delayed puberty noted. We recommend regularly evaluating pubertal state as part of the complete physical examination in ADA-SCID patients. If cryptorchidism is present, we suggest undertaking specialist urologic evaluation as soon as possible. Patients with cryptorchidism have an increased risk of progressive infertility, testicular malignancy, and torsion [8]; successful relocation of the testes may reduce these potential long-term sequelae. Considering the impact urogenital and pubertal abnormalities can have on patients' quality of life, we feel it is essential to include relevant history taking, clinical examination, and endocrine investigations in ADA-SCID patients to detect any abnormalities, initiate early treatment, and prevent long term complications.

Acknowledgments The Fondazione Telethon and San Raffaele Hospital developed gene therapy for ADA-SCID, for which GlaxoSmithKline (GSK) acquired their license. ADA-SCID gene therapy (Strimvelis) was licensed to GSK in 2010 and received European marketing authorization in 2016. These licenses were transferred to Orchard Therapeutics (OTL) in April 2018. AA and CB are the PIs of the ADA-SCID clinical trial for gene therapy.

All research at the Great Ormond Street Hospital NHS Foundation Trust and UCL Great Ormond Street Institute of Child Health is made possible by the NIHR Great Ormond Street Hospital Biomedical Research Centre. Several authors of this publication are members of the European Reference Network for Rare Immunodeficiency.

Funding Information Research at Great Ormond Street Hospital is supported by the NIHR GOSH Biomedical Research Centre. Funded by Fondazione Telethon (to AA); Ministero della Salute, Ricerca Finalizzata NET-2011-02350069 (to AA, CC, CA); Grant Ricerca Corrente Childrens' Hospital Bambino Gesù, Rome, Italy $201802 \mathrm{P} 004272$.

\section{Compliance with Ethical Standards}

Patients or their guardians provided written informed consent according to local consent procedures. This report was performed in accordance with the ethical standards of the institutional research committees and with the 1964 Helsinki declaration and its later amendments.

Disclaimer The views expressed are those of the author(s) and not necessarily those of the NHS, the NIHR, or the Department of Health. 
Open Access This article is licensed under a Creative Commons Attribution 4.0 International License, which permits use, sharing, adaptation, distribution and reproduction in any medium or format, as long as you give appropriate credit to the original author(s) and the source, provide a link to the Creative Commons licence, and indicate if changes were made. The images or other third party material in this article are included in the article's Creative Commons licence, unless indicated otherwise in a credit line to the material. If material is not included in the article's Creative Commons licence and your intended use is not permitted by statutory regulation or exceeds the permitted use, you will need to obtain permission directly from the copyright holder. To view a copy of this licence, visit http://creativecommons.org/licenses/by/4.0/.

\section{References}

1. Bradford KL, Moretti FA, Carbonaro-Sarracino DA, Gaspar HB, Kohn DB. Adenosine deaminase (ADA)-deficient severe combined immune deficiency (SCID): molecular pathogenesis and clinical manifestations. J Clin Immunol. 2017;37(7):626-37. https://doi. org/10.1007/s10875-017-0433-3.

2. Kohn DB, Hershfield MS, Puck JM, Aiuti A, Blincoe A, Gaspar $\mathrm{HB}$, et al. Consensus approach for the management of severe combined immune deficiency caused by adenosine deaminase deficiency. J Allergy Clin Immunol. 2019;143(3):852-63. https://doi.org/ 10.1016/j.jaci.2018.08.024.

3. Whitmore KV, Gaspar HB. Adenosine deaminase deficiency - more than just an immunodeficiency. Front Immunol. 2016;7:314. https://doi.org/10.3389/fimmu.2016.00314.

4. Flinn AM, Gennery AR. Adenosine deaminase deficiency: a review. Orphanet J Rare Dis. 2018;13(1):65. https://doi.org/10. 1186/s13023-018-0807-5.

5. Scott O, Kim VH, Reid B, Pham-Huy A, Atkinson AR, Aiuti A, et al. Long-term outcome of adenosine deaminase-deficient patients-a single-center experience. Clin Immunol. 2017;37(6):58291. https://doi.org/10.1007/s10875-017-0421-7.

6. Holland AJ, Nassar N, Schneuer FJ. Undescended testes: an update. Curr Opin Pediatr. 2016;28(3):388-94. https://doi.org/10.1097/ MOP.0000000000000335.

7. Hutson JM. Cryptorchidism and hypospadias. In: Endotext [Internet]. South Dartmouth (MA): MDText.com, Inc.; 20002018. https://www.ncbi.nlm.nih.gov/books/NBK279106/ Bookshelf ID: NBK279106. Accessed 10 July 2018.
8. Keys C, Heloury Y. Retractile testes: a review of the current literature. J Pediatr Urol. 2012;8(1):2-6. https://doi.org/10.1016/j.jpurol. 2011.03.016.

9. Salonia A, Rastrelli G, Hackett G, Seminara SB, Huhtaniemi IT, Rey RA, et al. Paediatric and adult-onset male hypogonadism. Nat Rev Dis Primers. 2019;5(1):38. https://doi.org/10.1038/s41572019-0087-y.

10. Blackburn MR, Kellems RE. Adenosine deaminase deficiency: metabolic basis of immune deficiency and pulmonary inflammation. Adv Immunol. 2005;86:1-41. https://doi.org/10.1016/S00652776(04)86001-2.

11. Ferrari D, Gambari R, Idzko M, Müller T, Albanesi C, Pastore S, et al. Purinergic signaling in scarring. FASEB J. 2016;30(1):3-12. https://doi.org/10.1096/fj.15-274563.

12. Dalle JH, Lucchini G, Balduzzi A, Ifversen M, Jahnukainen K, Macklon KT, et al. State-of-the-art fertility preservation in children and adolescents undergoing haematopoieticstem cell transplantation: a report on the expert meeting of the Paediatric Diseases Working Party (PDWP) of the European Society for Blood and Marrow Transplantation (EBMT) in Baden, Austria, 29-30 September 2015. Bone Marrow Transplant. 2017;52(7):1029-35. https://doi.org/10.1038/bmt.2017.21.

13. Gennery AR, Lankester A. Long term outcome and immune function after hematopoietic stem cell transplantation for primary immunodeficiency. Front Pediatr. 2019;7:381. https://doi.org/10. 3389/fped.2019.00381 eCollection 2019.

14. Lum SH, Flood T, Hambleton S, McNaughton P, Watson H, Abinun M, et al. Two decades of excellent transplant survival in children with chronic granulomatous disease: a report from a supraregional immunology transplant center in Europe. Blood. 2019;133(23):2546-9. https://doi.org/10.1182/blood.2019000021.

15. Faraci M, Diesch T, Labopin M, Dalissier A, Lankester A, Gennery A, et al. Gonadal function after Busulphan compared to Treosulphan in children and adolescents undergoing allogeneic haematopoietic stem cell transplantation. On Behalf of Pediatric and Transplant-Related Complications and Quality of Life EBMT Working Parties. Biol Blood Marrow Transplant. 2019;25(9):178691. https://doi.org/10.1016/j.bbmt.2019.05.005.

16. Mi T, Abbasi S, Zhang H, Uray K, Chunn JL, Xia LW, et al. Excess adenosine in murine penile erectile tissues contributes to priapism via A2B adenosine receptor signaling. J Clin Invest. 2008;118(4): 1491-501. https://doi.org/10.1172/JCI33467.

Publisher's Note Springer Nature remains neutral with regard to jurisdictional claims in published maps and institutional affiliations. 